\title{
AN ASYMPTOTIC FORMULA FOR THE EIGENVALUES OF THE LAPLACIAN OPERATOR IN AN UNBOUNDED DOMAIN
}

\author{
BY COLIN CLARK ${ }^{1}$
}

Communicated by F. Browder, February 10, 1966

F. Rellich [5] and, more generally, A. M. Molcanov [3] have shown that the problem

$$
\begin{aligned}
\frac{1}{2} \Delta^{2} u(x)+\lambda u(x) & =0, & & x \in \Omega \\
u(x) & =0, & & x \in \partial \Omega
\end{aligned}
$$

has a discrete spectrum (and consequently a complete orthonormal system of eigenfunctions in $\mathscr{L}_{2}(\Omega)$ ) provided that $\Omega$ is a "quasibounded" domain in $E_{n}$. A domain $\Omega$ is said to be quasi-bounded if it is either bounded or satisfies

$$
\lim _{x \rightarrow \infty, x \in \Omega} \operatorname{dist}(x, \partial \Omega)=0 .
$$

(See [1] for a proof of Molcanov's result, based on a generalization of the Kondrachoff embedding theorem for the Sobolev spaces $H_{0}^{m}(\Omega)$.) The problem of determining the asymptotic behavior of the eigenvalues of (1) has remained open (cf. [2, p. 233]).

In the present note we consider the above problem from the point of view of random processes, as described in detail for the case of a bounded domain, as well as for the case of the operator $-\frac{1}{2} \Delta^{2}+V(x)$ (with $V(x) \rightarrow+\infty$ as $|x| \rightarrow \infty$ ) on an unbounded domain, in the papers of D. Ray [4] and M. Rosenblatt [6]. We will show that if $\Omega$ satisfies the following condition

$$
m(\Omega \cap[a<|x|<a+1])=O\left(a^{-\beta}\right)
$$

for some $\beta>\frac{1}{2}$, then simple modifications of Ray's arguments suffice to prove discreteness of the spectrum, as well as to obtain an asymptotic formula for the eigenvalues.

We take Ray's paper [4] as a starting point. Thus (assuming a cone condition for $\Omega$, as described in Theorem 1 below) we already have a Green's function $K(x, y, t)$ corresponding to the equation

\footnotetext{
1 Research supported in part by the United States Air Force Office of Scientific Research, grant number AF-AFOSR-379-65.
} 


$$
\Delta^{2} \phi(x, t)=2 \frac{\partial}{\partial t} \phi(x, t), \quad x \in \Omega, \quad t>0
$$

and zero boundary conditions. We first wish to verify that the integral operator $K_{t}$ with kernel $K(x, y, t)$ is completely continuous in $\mathscr{L}_{2}(\Omega)$. As in [4, Lemma 3$]$ we see that it is sufficient to show, for fixed $t>0$,

$$
\int_{\Omega \cap[|x|>a]}\left|K_{t} \psi(x)\right|^{2} d x \rightarrow 0 \quad \text { as } a \rightarrow \infty,
$$

uniformly for $\psi \in \mathfrak{L}_{2}(\Omega),\|\psi\|=1$. But, as in [4],

$$
\begin{aligned}
\int_{\Omega \cap[|x|>a]}\left|K_{t} \psi(x)\right|^{2} d x & \\
\leqq & \int_{\Omega \cap[|x|>a]} d x \cdot \operatorname{prob}\{x+x(\tau) \in \bar{\Omega}, 0 \leqq \tau \leqq t\} \cdot\|\psi\|^{2} .
\end{aligned}
$$

By an elementary calculation using (2), we have for any $\beta^{\prime}<\beta$

$$
\begin{aligned}
\operatorname{prob}\{x+x(\tau) \in \bar{\Omega}, 0 & \leqq \tau \leqq t\} \\
& \leqq \operatorname{prob}\{x+x(t) \in \bar{\Omega}\}=O\left(|x|-\beta^{\prime}\right), \quad x \in \Omega ;
\end{aligned}
$$

here $t$ is fixed. Hence, writing $\Omega_{i}=\Omega \cap[i \leqq|x|<i+1], i=0,1,2, \cdots$, we have (taking $\beta^{\prime}>\frac{1}{2}$ )

$$
\begin{aligned}
& \int_{\Omega} \operatorname{prob}\{x+x(\tau) \in \bar{\Omega}, 0 \leqq \tau \leqq t\} d x \\
&=\sum_{i} \int_{\Omega_{i}} \operatorname{prob}\{x+x(\tau) \in \bar{\Omega}, 0 \leqq \tau \leqq t\} d x \\
&=O\left(\sum_{i} i^{-2 \beta^{\prime}}\right)<\infty,
\end{aligned}
$$

and (4) follows from this. We therefore have

THEOREM 1. Let $\Omega$ be an open set in $E_{n}$, satisfying condition (2) and the following cone condition: for each $x \in \partial \Omega$ there is an open cone with vertex $x$, lying outside $\bar{\Omega}$. Let $K_{t}$ be the integral operator in $\mathfrak{L}_{2}(\Omega)$ with kernel $K(x, y, t)$.

Then $K_{t}$ is completely continuous and hence has a countable set of eigenvalues $\left\{\exp \left(-\lambda_{j} t\right), j=0,1,2, \cdots\right\}$ with corresponding complete orthonormal eigenfunctions $\left\{\phi_{j}(x)\right\}$, which are independent of $t$. Moreover the $\lambda_{j}$ are eigenvalues and the $\phi_{j}$ eigenfunctions of the problem (1). 
Corollary. Let $\Omega$ be as in Theorem 1. Then

$$
\sum_{\lambda_{j}<\lambda} \phi_{j}^{2}(x) \sim\left(\frac{\lambda}{2 \pi}\right)^{n / 2} \cdot \frac{1}{\Gamma(1+n / 2)}
$$

as $\lambda \rightarrow \infty$, for each $x \in \Omega$.

The proofs of the asserted properties of the $\lambda_{j}$ and $\phi_{j}$ are the same as in Ray's paper. In particular, Ray shows that

$$
\sum_{j} \exp \left(-\lambda_{j} t\right) \phi_{j}^{2}(x)=K(x, x, t) \sim\left(\frac{1}{2 \pi t}\right)^{n / 2}
$$

as $t \rightarrow 0$, uniformly for $x \in \Omega$; in the present case this follows from the fact that $K(x, y, t)$ is a Hilbert-Schmidt kernel, as can be proved in a manner similar to the above verification of (4)-it is useful to notice that $0 \leqq K(x, y, t) \leqq(2 \pi t)^{-n / 2} \cdot \exp \left(-|x-y|^{2} / 2 t\right)$.

THEOREM 2. Let $\Omega \subset E_{n}$ satisfy the hypotheses of Theorem 1. Let $\rho(x)$ be a nonnegative function in $\mathfrak{L}_{1}(\Omega)$. Define

$$
N_{\rho}(\lambda)=\sum_{\lambda_{j} \leqq \lambda} \int_{\Omega} \rho(x) \phi_{j}^{2}(x) d x .
$$

Then

$$
N_{\rho}(\lambda) \sim\left(\frac{\lambda}{2 \pi}\right)^{n / 2} \frac{1}{\Gamma(1+n / 2)} \int_{\Omega} \rho(x) d x .
$$

Proof. Applying (5) to the Laplace-Stieltjes transform of $N_{\rho}(\lambda)$, we have

$$
\begin{aligned}
\int_{0}^{\infty} e^{-\lambda t} d N_{\rho}(\lambda) & =\int_{\Omega} \rho(x) \sum_{j} \exp \left(-\lambda_{j} t\right) \phi_{j}^{2}(x) d x \\
& =\int_{\Omega} \rho(x) K(x, x, t) d x \\
& \sim\left(\frac{1}{2 \pi t}\right)^{n / 2} \int_{\Omega} \rho(x) d x
\end{aligned}
$$

Hence the Tauberian theorem of Karamata applies, and yields (6). q.e.d.

We obviously obtain the classical formula of Weyl if we put $\rho(x) \equiv 1$ on a bounded region (or even on an unbounded region of finite volume). If in the general case we choose a bounded function $\rho(x)$, we obtain $N_{\rho}(\lambda) \leqq c \cdot N(\lambda)$ where $N(\lambda)=N_{1}(\lambda)$ is the usual function; we 
therefore obtain a one-sided estimate for $N(\lambda)$ :

$$
N(\lambda) \succ \lambda^{n / 2}
$$

where $f(\lambda) \succ g(\lambda)$ means the same as $g(\lambda)=O(f(\lambda))$. We remark that our results are unaffected if the operator $-\Delta^{2}$ is replaced by $-\Delta^{2}$ $+V(x)$ if $V(x)$ is a bounded function on $\Omega$.

The foregoing results can also be derived using analytical methods similar to those of Titchmarsh [7]; the basic properties of the Green's function $G(x, y, \lambda)$ in this case are due to D. Hewgill (Thesis, University of British Columbia).

\section{REFERENCES}

1. Colin Clark, An embedding theorem for function spaces, Pacific J. Math. (to appear)

2. I. M. Glazman, Prijamye metody kačestvennovo spectral'novo analiza singuljarnyh differencial'novo operatorov, Fizmatgiz, Moscow, 1963.

3. A. M. Molcanov, On conditions for the discreteness of the spectrum of a differential operator of second order (Russian), Trudy Moskov. Mat. Obšc. 2 (1953), 169-200.

4. Daniel Ray, On the spectra of second order differential operators, Trans. Amer. Math. Soc. 77 (1954), 299-321.

5. F. Rellich, Das Eigenwertproblem von $\Delta u+\lambda u=0$ in Halbröhren, in Essays presented to R. Courant, Interscience, New York, 1948, 329-344.

6. M. Rosenblatt, On a class of Markov processes, Trans. Amer. Math. Soc. 71 (1951), 120-135.

7. E. C. Titchmarsh, Eigenfunction expansions associated with second order differential equations, Part II, Oxford Univ. Press, 1958.

The University of British Columbia 